\title{
REQUISITOS E CONCEITO DE UM APLICATIVO PARA O CENTRO DE REFERÊNCIA EM SÍNDROME DE DOWN (CRISDOWN)
}

Angelica Aguiar Nunes Resende (resende.angelica@hotmail.com) - Universidade de Brasília

Sanderson Barbalho (도bbr@yahoo.com.br) - Universidade de Brasília

Elaine Silva (elainecps.br@gmail.com) - Departamento de Engenharia Mecânica / Faculdade de Tecnologia / Universidade de Brasília

\section{RESUMO}

O desenvolvimento de produto envolve diversas fases para atingir a estratégia, realizando o levantamento e compreendendo as necessidades, para que a entrega atinja às expectativas e atenda às restrições do cliente. Neste intuito, o presente estudo tem como objetivo apresentar o desenvolvimento de requisitos e o conceito de um aplicativo que auxilie na gestão do Centro de Referência Interdisciplinar em Síndrome de Down - Crisdown, localizado em Brasília - DF, considerando as necessidades e expectativas do cliente e as restrições do projeto. O método aplicado derivou das etapas iniciais de modelos de referências consagrados em desenvolvimento de produto, passando por algumas adaptações tendo em vista que o produto é um aplicativo. O artigo inclui as especificações do produto, o conceito do produto e o plano de negócios.

Palavras chave: Síndrome de down; Processo de desenvolvimento de produtos; Aplicativo; Requisitos; Conceito do produto 


\section{INTRODUÇÃO}

A Síndrome de Down - SD é uma condição de vida advinda da presença de 47 cromossomos nas células em vez de 46. O indivíduo com a Síndrome de Down possui cromossomos normais. Porém, no par 21, há um cromossomo extra. A comunidade científica considera três tipos de síndrome de Down: a trissomia 21 (95\% dos casos), mosaico e translocação (DÉA e DÉA, 2009).

A Síndrome de Down, por décadas, foi tratada como condição de inferioridade. Apesar de atualmente o conhecimento acumulado e as informações estarem mais acessíveis, o estigma ainda está presente e influencia na imagem e na reação dos pais em relação à criança com SD (VOIVODIC e STORER, 2002).

Sabe-se que acreditar na capacidade, oferecer estimulação, amor, educação e respeito são os ingredientes para que a pessoa com síndrome de Down tenha todas as condições de se tornar um adulto capaz, produtivo e feliz (DÉA e DÉA, 2009).

Diante do conhecimento explicitado acima, em abril de 2013, o Hospital Regional da Asa Norte, localizado em Brasília - DF, inaugurou o Centro de Referência Interdisciplinar em Síndrome de Down - Crisdown, um centro de tratamento especializado para pessoas com Síndrome de Down. O Crisdown é referência de qualidade e engajamento na luta pelo desenvolvimento e inclusão de pessoas com síndrome de Down. Seu atendimento parte da necessidade de identificar quem são os pacientes com SD, centralizando, inicialmente, por meio de palestra de acolhimento.

Após a palestra, os pacientes são direcionados aos médicos do Crisdown que identificam os problemas e quais acompanhamentos são necessários. Dependendo da avaliação, o caso é direcionado a profissionais que atendam o mais próximo da residência do paciente. Desta forma, a probabilidade de evasão do tratamento é minimizada. Mesmo assim, os pacientes precisam retornar periodicamente ao Crisdown para o acompanhamento da evolução e identificação dos problemas que necessitam de maior atenção.

O Centro de Referência em Síndrome de Down passou a ser mais conhecido e sua demanda vem crescendo, porém, a quantidade de profissionais ainda é limitada para atender a todos que aguardam na fila de espera. Atualmente, a prioridade do atendimento ocorre de acordo com análise subjetiva do médico, que considera o estado de saúde e familiar do paciente. Porém, é nítida a necessidade do Crisdown de padronizar os critérios para a classificação de riscos ambulatoriais, de modo a maximizar o atendimento e tratar com urgência os casos considerados mais graves.

Diante do exposto, o presente estudo buscará desenvolver requisitos e conceito para um aplicativo que consiga atender as necessidades dos stakeholders do Crisdown. Visando facilitar o trabalho dos profissionais, no que diz respeito às atividades administrativas (contato com pacientes, marcação de 
consultas e atividades, registro de novos pacientes, localização dos registros e históricos) e a classificação da fila de espera.

O objetivo deste aplicativo ultrapassa as funcionalidades de um prontuário eletrônico, pois trata-se de viabilizar a aplicação da Linha do cuidado a pessoas com Síndrome de Down. Linha do cuidado são fluxos assistenciais à saúde que conduzem os processos de trabalho (cuidados) de forma eficiente e eficaz, distribuindo os serviços ofertados.

O aplicativo proposto está em desenvolvimento e sua concepção foi realizada com base nas metodologias de projeto de produto, utilizando modelos de referência em desenvolvimento de produtos adicionados de uma maior sistemática de elucidação e consolidação de requisitos de software. $\mathrm{O}$ presente artigo apresenta essa etapa do trabalho. Na seção seguinte são apresentados o referencial teórico básico da pesquisa. Em seguida, apresenta-se a metodologia do trabalho, os resultados e considerações finais.

\section{REVISÃO TEÓRICA}

\subsection{PROCESSO DE DESENVOLVIMENTO DE PRODUTOS}

Segundo Rozenfeld et al. (2006), o desenvolvimento de produtos consiste em um conjunto de atividades que buscam, a partir das necessidades do mercado e das possibilidades e restrições tecnológicas, chegar às especificações de um produto e ao seu processo de produção, pensando sempre na capacidade de produzi-lo. Além disso, é necessário considerar as estratégias competitivas e de produto da empresa.

Para viabilizar o PDP, é necessário adotar uma metodologia que indique o caminho a ser percorrido. Desta forma, traz-se o modelo adotado por Rozenfeld (2006), ilustrado na Figura 1, onde demonstra a divisão em macrofases, fases e atividades. 
Visāo geral do modelo de referência adotado neste livro.

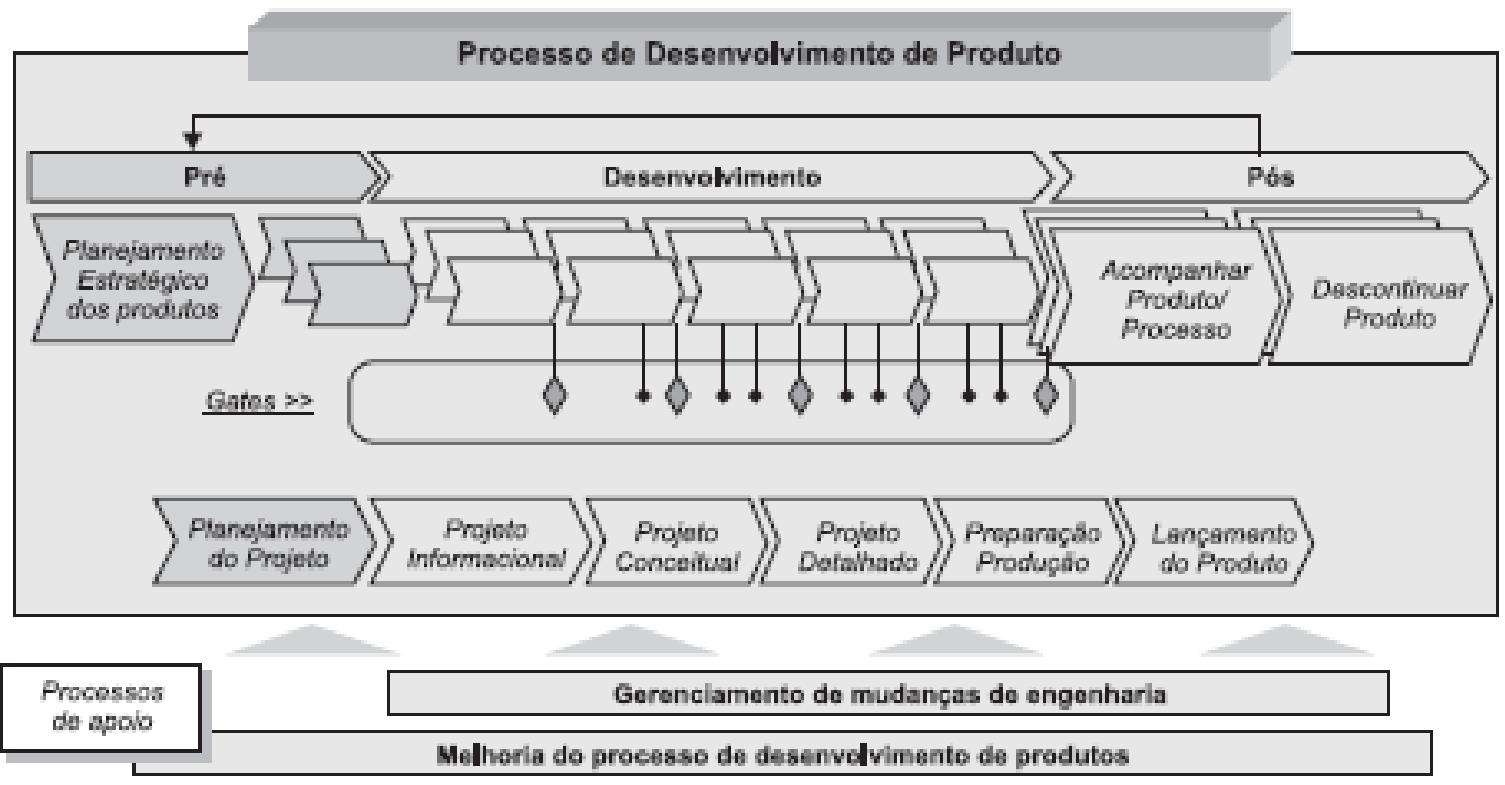

Figura 1- Visão geral do modelo de referência

Fonte: Rozenfeld (2006)

As macrofases são:

a. Pré-desenvolvimento: composta pelas fases de Planejamento Estratégico do Produto e Planejamento do Projeto.

i. Como resultado, obtém-se dois documentos: portfólio de produtos e minuta do projeto.

ii. O resultado da fase Planejamento do Projeto é o plano do projeto.

b. Desenvolvimento: composta pelas fases de Projeto Informacional, Projeto Conceitual, Projeto Detalhado, Preparação da Produção e Lançamento do Produto.

i. O resultado do Projeto Informacional é a especificação-meta do futuro produto.

ii. O resultado do Projeto Conceitual é a concepção do produto.

iii. No Projeto Detalhado, a concepção do produto é detalhada e transformada em Especificações finais, após aprovadas são congeladas.

iv. Preparação da Produção: o produto é certificado e é gerada a liberação da produção.

v. Lançamento do Produto: finalizado na emissão do documento oficial de lançamento. 
c. Pós-desenvolvimento: composta pelas fases de acompanhar produto/processo e descontinuar produto.

Tratando-se ainda a respeito de metodologia, traz-se também o conceito do Modelo de Referência Mecatrônico - MRM (Barbalho, 2006), representado pela Figura 2, voltado para desenvolvimento de produtos mecatrônicos que reflete as melhores práticas destes.

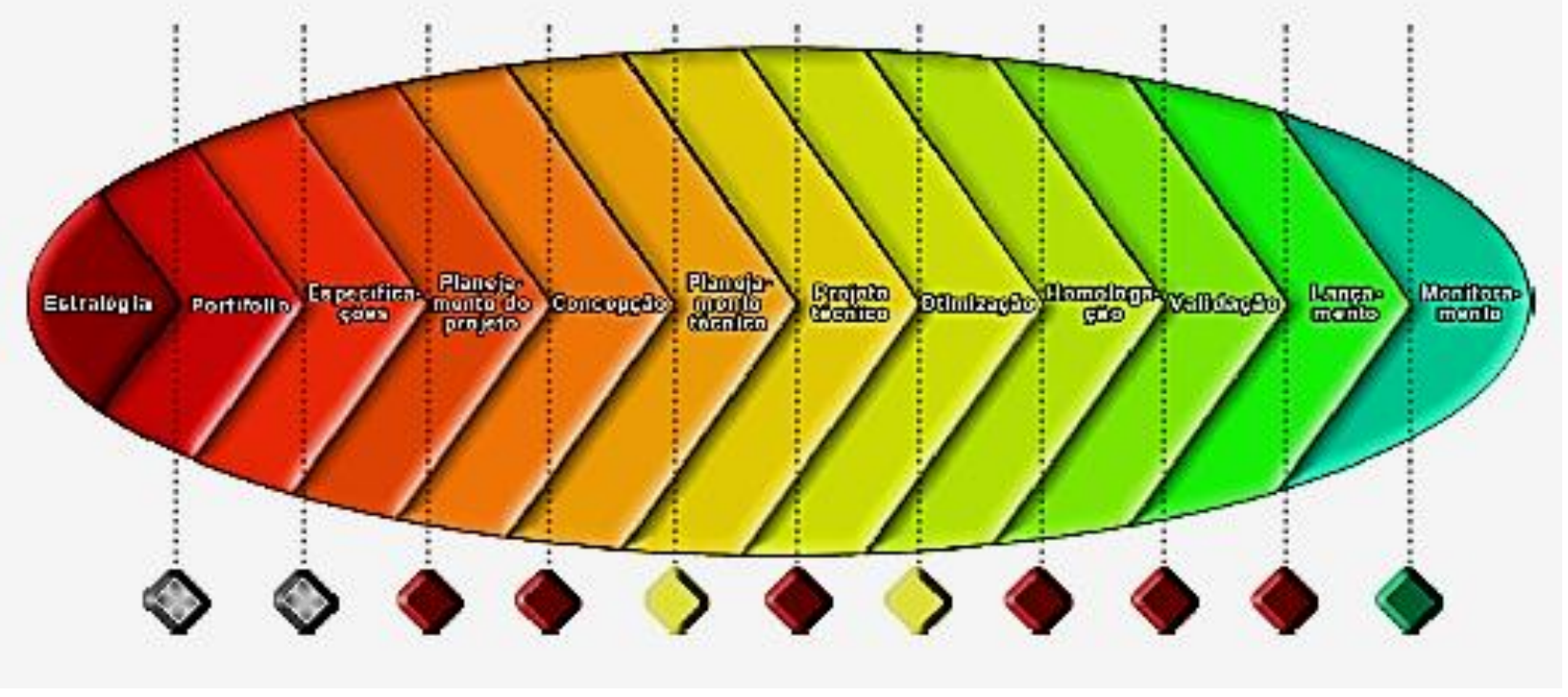

Figura 2- Fases do MRM com estrutura de decisões do PDP

Fonte: Barbalho e Rozenfeld (2013)

A Figura 2 trata de um modelo em fases. Baseado em cadeia de agregação de valor. Abaixo o resumo das fases do MRM, de acordo com Barbalho (2006):

a. Estratégia: definição dos objetivos estratégicos a serem perseguidos em cada linha de produtos (LDP).

b. Portfólio: definição do portfólio de cada LDP. Quanto à decisão, versa sobre uma linha de produtos.

c. Especificações: definiçãa da especificação de cada produto.

d. Planejamento do projeto: definição do plano do projeto. Decisão go/kill relacionada com a viabilidade dos prazos estimados para ele e os custos decorrentes.

e. Concepção: definição dos principais componentes, bem como princípios de solução para as funções principais do produto mecatrônico.

f. Planejamento técnico: detalhamento do plano do projeto com base na concepção definida no qual constam modelos funcionais e de arquitetura do produto. 
g. Projeto técnico: soluções técnicas para as funções principais do produto.

h. Otimização: detalhamento e teste de soluções para funções secundárias do produto e realização de análises necessárias ao aumento da robustez e confiabilidade do produto.

i. Homologação: homologação do processo de fabricação e montagem do produto.

j. Validação: consiste em validação e certificação do produto.

k. Lançamento: lançamento do produto no mercado.

1. Monitoramento: acompanhamento dos resultados conseguidos com o produto e gerenciamento das modificações realizadas na configuração inicial de produção.

Esses modelos aqui sumarizados foram a base para o desenvolvimento do trabalho, no qual desenvolveu-se um detalhamento maior da fase de especificações em que os requisitos e o conceito do produto são consolidados.

\subsection{ENGENHARIA DE REQUISITOS}

Segundo Pressman e Maxin (2016), a engenharia de requisitos é uma ação da engenharia de software importante que se inicia durante a atividade de comunicação e continua na modelagem. Esta atividade deve ser adaptada às necessidades do processo, do projeto, do produto e das pessoas que estão realizando o trabalho. A engenharia de requisitos estabelece uma base sólida para o projeto e construção. Sem ela, o software resultante tem grande probabilidade de não atender às necessidades do cliente.

Necessidades dos clientes, segundo Rozenfeld (2006), são dados originais dos desejos dos clientes, que podem ser redundantes e expressar características dos produtos. Dessa forma, deve-se buscar levantar as necessidades dos clientes de cada fase do ciclo de vida do produto. Essas necessidades podem ser obtidas com o uso de listas de verificação, por meio da observação direta, entrevistas, entre outros diversos métodos.

Segundo Zave (1997), a engenharia de requisitos é a área da engenharia de software que se preocupa com as metas reais, funções e restrições de software. Ela busca o relacionamento destes fatores a uma precisa especificação do comportamento do software e sua evolução através do tempo e das famílias.

Pressman e Maxin (2016) salienta que a ampla disponibilidade de aplicativos no mercado eletrônico levará a requisitos mais diversificados. Os stakeholders podem personalizar um produto na intenção cumprir requisitos específicos para atender um pequeno grupo de usuários. 
Desta forma, os ciclos de desenvolvimento são reduzidos, aumentando a pressão para otimizar a engenharia de requisitos e possibilitar que os produtos cheguem ao mercado mais rapidamente.

Sommerville (2007) conceitualiza os requisitos funcionais e não funcionais de um sistema:

i. Requisitos funcionais: São as declarações de serviços que o sistema deve fornecer, como o sistema deve reagir a entradas específicas e como deve se comportar em determinadas situações. Às vezes pode estabelecer o que o sistema não deve fazer.

ii. Requisitos não funcionais: São restrições sobre os serviços ou funções oferecidas pelo sistema. Aplicam-se ao sistema como um todo e geralmente se adéquam às características ou serviços individuais de sistema.

No presente trabalho, utiliza-se essa classificação de requisitos funcionais e não-funcionais para sistematizar as especificações do aplicativo.

\section{METODOLOGIA}

Segundo Barbalho (2006), modelo é uma representação externa e explícita. Rozenfeld (2006) explicita o modelo unificado do Processo de Desenvolvimento de Produtos (PDP), que significa descrever as atividades, recursos, informações, fases, responsabilidades e outras possíveis dimensões do processo.

Neste sentido, foram realizadas adaptações e combinações dos modelos supramencionados, melhor demonstrados na Figura 2 e na Figura 3. Assim, o presente estudo, considerou as fases iniciais para a formulação da metodologia.

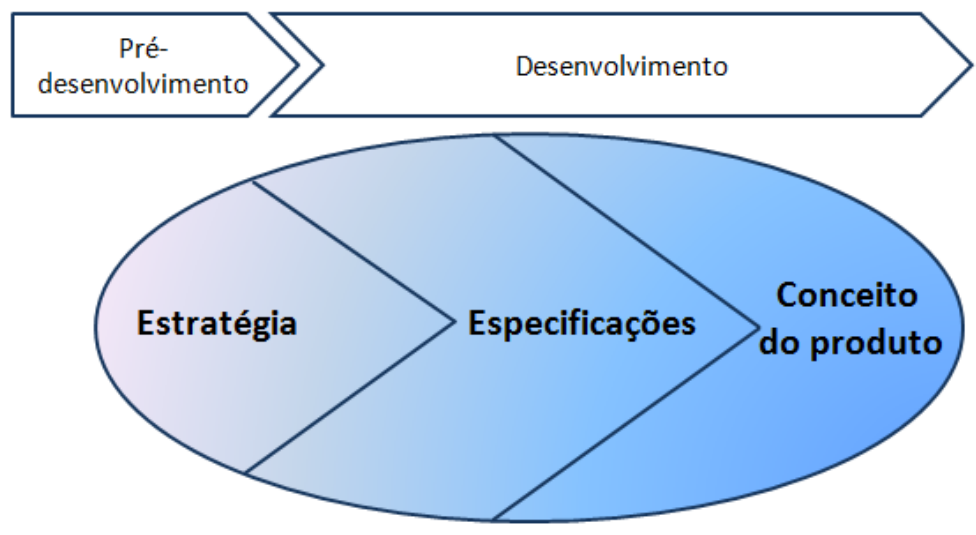

Figura 3 - Modelo de desenvolvimento da proposta 
Fonte: Elaborado pela autora

A Figura 3 demonstra as duas etapas principais do projeto, que são o Pré-desenvolvimento e o Desenvolvimento. Na primeira etapa, serão trabalhados os conceitos: a Estratégia, proposta por Barbalho (2006), a qual, entretanto, foge ao escopo do presente artigo. Na segunda etapa, serão trabalhados as especificações e o conceito do produto por Barbalho (2006). A fase de portfólio não é tratada neste trabalho, uma vez que o objetivo é voltado a um aplicativo computacional. Para a etapa de requisitos, foco desse trabalho, utiliza-se o esquema ilustrado na Figura 4, contempla a fase do Projeto informacional de Rozenfeld (2006) e a fase de Especificações do produto de Barbalho (2006). Por se tratar de aplicativo, nesta fase deve ser iniciada a arquitetura do sistema, que justamente trata da gestão da especificação e seu levantamento.

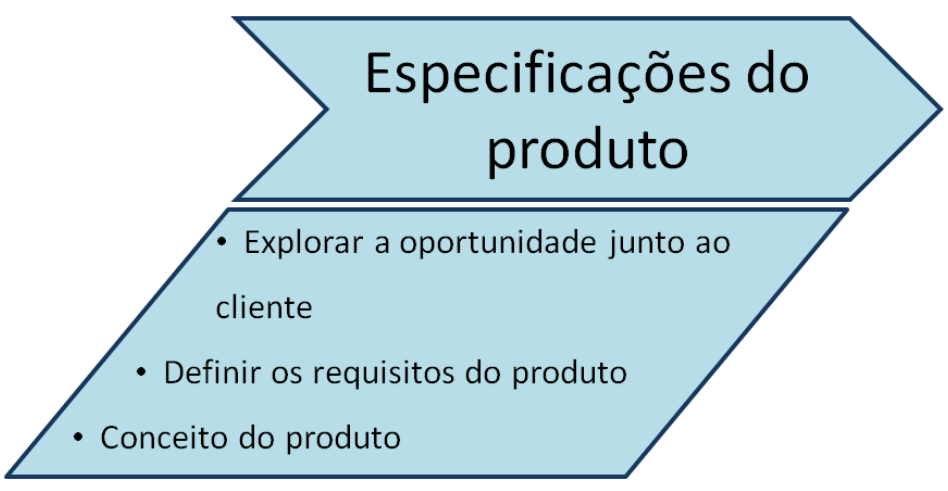

Figura 4- Projeto Informacional

Fonte: Elaborado pela autora

Para o desenvolvimento desta fase, foram realizadas as três atividades a seguir:

- Explorar a oportunidade junto ao cliente: Levantar informações que auxiliam na compreensão da real necessidade dos potenciais usuários, de forma a perceber suas expectativas com o projeto e percepção em relação a outros aplicativos.

- Definir os requisitos do produto: A partir do levantamento com os clientes e das demais análises, serão descritos alguns requisitos técnicos do produto, como por exemplo, suas funcionalidades, quais dispositivos utilizar, entre outros.

- Conceito do produto: Definir o conceito do produto utilizando as informações levantadas dos requisitos do produto e demais análises já realizadas. 


\section{RESULTADOS}

\subsection{REQUISITOS E NECESSIDADES DOS CLIENTES}

Foram realizadas diversas reuniões com o cliente do projeto, bem como visitas ao Crisdown para entender o ambiente e suas necessidades. Nas reuniões foi possível extrair as necessidades do Centro de Referência em Síndrome de Down - Crisdown, além das necessidades pais e familiares que acompanham seus filhos ao Crisdown, tanto para o acolhimento, quanto para as consultas ou outras atividades.

Acolhimento é um termo utilizado para as reuniões que acontecem mensalmente para receber novos pacientes com Síndrome de Down. O dia do acolhimento infantil é diferente do dia do acolhimento adulto. Tendo em vista que as fases de vida são diferentes, tanto o foco do acolhimento quanto à estimulação deve ocorrer em acordo com estas fases, para assim preparar os pacientes para situações enfrentadas no cotidiano.

O foco principal deste produto é atender as demandas do Crisdown, tendo em vista que o sistema utilizado no hospital, fornecido pelo governo do Distrito Federal, não disponibiliza os recursos necessários para colocar em prática a Linha do Cuidado aplicada a pessoas com Síndrome de Down.

\subsection{DEFINIÇÃO DOS REQUISITOS DO PRODUTO}

Segundo a equipe do Crisdown, o acolhimento constitui o primeiro contato da família e do paciente com a equipe. Nesse encontro, é ministrada uma palestra sobre as principais informações relacionadas à Síndrome de Down e ao Crisdown.

No acolhimento são coletados os dados dos pacientes. Dependendo da quantidade de pessoas, os pacientes são atendidos pelo médico no mesmo dia. Caso não seja possível, a consulta é marcada. Nos acolhimentos, nota-se que, pelos relatos, há falta de informações aos pais de crianças com SD, sobretudo daqueles que recentemente descobriram a síndrome. Outro fator citado, é o desconhecimento do público em relação à existência do Centro de Referência direcionado a pessoas com SD.

Percebeu-se que muitos registros eram realizados manualmente, e só então eram repassados às planilhas e ao sistema do governo. Em conversa com a equipe do Crisdown ficaram evidentes 
as dificuldades em preencher fichas manuais para depois digitar nas planilhas e no sistema do governo. Essa ação gera "retrabalho", uma vez que a equipe necessitará de mais tempo para realizar atividades burocráticas, que poderiam ser minimizadas caso existisse um sistema específico para o Crisdown.

O sistema utilizado pelo governo do Distrito Federal - DF foi desenvolvido para atender as necessidades de consulta e outras rotinas existentes em hospital e clínica, porém não considera as especificidades existentes nos diversos casos atendidos na rede pública de saúde. No caso do Crisdown, o sistema utilizado não atende forma satisfatória, pois há demandas específicas que o sistema não consegue atender.

Informações significativas para o tratamento de crianças e adultos com SD ficam registradas no campo de "observações", quando na verdade, deveriam, pela importância e relevância, estar em campos específicos, facilitando assim a busca pela informação.

Diante da situação exposta, fica evidente a necessidade do Crisdown de possuir um sistema personalizado que atenda aos seus interesses. Não obstante, é importante salientar que o sistema busque viabilizar a linha do cuidado, para que esses pacientes sejam devidamente acompanhados e que suas famílias tenham a orientação necessária para o prosseguimento do tratamento.

Nas reuniões, a equipe explicitou as necessidades pertinentes ao centro de referência, bem como dos profissionais de saúde que integram a equipe e dos familiares da pessoa com SD. No Quadro 1, são apresentadas as necessidades do cliente identificadas. 
Quadro 1 - Necessidades do cliente

\begin{tabular}{|c|c|}
\hline Necessidades do Cliente & Requisitos dos Clientes \\
\hline Qualquer usuário precisa mexer sem dificuldades & \multirow{3}{*}{ Ser de fácil manuseio } \\
\hline A inserção de dados deve ser fácil & \\
\hline Realizar consultas facilmente & \\
\hline As informações têm que estar disponíveis a qualquer tempo & Ser disponível \\
\hline Os formulários devem ser padronizados & \multirow{2}{*}{ Ser padronizado } \\
\hline Tem que ter campo para condição de saúde & \\
\hline Diferenciar cada especialidade em sua fila de espera & \multirow{3}{*}{ Ser preciso } \\
\hline Tem que identificar as especialidades mais urgentes & \\
\hline Tem que ordenar a fila de espera & \\
\hline As consultas devem ser rápidas & \multirow{2}{*}{ Ser rápido } \\
\hline A alteração e/ou o salvamento de dados deve ser rápido & \\
\hline Apenas os médicos do Crisdown podem ter acesso a todas as informações dos pacientes & \multirow{2}{*}{ Ser seguro } \\
\hline As informações devem estar seguras & \\
\hline As informações inseridas devem ser objetivas & \multirow{2}{*}{ Ter campos e caracteres limitados } \\
\hline O lançamento das informações no aplicativo não deve significar retrabalho & \\
\hline Preencher facilmente os formulários & Ter tabelas de dados pré-definidas \\
\hline
\end{tabular}

Fonte: Elaborado pela autora

Definidos os requisitos do cliente, torna-se fundamental identificar os requisitos do sistema, funcionais e não-funcionais 0 . No Quadro 1 são apresentados os requisitos funcionais identificados e no Quadro 2 os não funcionais.

Os requisitos funcionais e não-funcionais também são baseados nas informações prestadas pelo cliente. Desta forma foi possível obter diversas informações necessárias para o desenvolvimento do aplicativo. 
Quadro 2 - Requisitos funcionais

\begin{tabular}{|c|c|}
\hline \multicolumn{2}{|r|}{ Requisitos funcionais identificados } \\
\hline Acesso & a autenticação deve ocorrer por meio de login. \\
\hline Nível de acesso & $\begin{array}{l}\text { as informações a serem acessadas dependem do perfil do usuário. Se for paciente ou responsável, terá acesso } \\
\text { apenas às informações do paciente que acessou. Se for profissional de saúde, terá acesso a algumas informações. Se } \\
\text { for médico do Crisdown, terá acesso a todos os dados. }\end{array}$ \\
\hline \multirow[t]{2}{*}{ Cadastro } & $\begin{array}{l}\text { 1. o cadastramento deve ocorrer no próprio aplicativo, quando o perfil for de profissional de saúde e médico do } \\
\text { Crisdown, o administrador do aplicativo tem que verificar e confirmar esta informação. }\end{array}$ \\
\hline & 2. O usuário deve poder se descadastrar do aplicativo quando quiser. \\
\hline \multirow{3}{*}{ Espaços } & 1. O aplicativo deve oferecer espaço para fóruns e discussões. \\
\hline & 2. deve contar espaço para direcionar mensagem aos médicos do Crisdown. \\
\hline & 3. Deve conter espaço para interessados em atender pacientes de SD se registrem. \\
\hline \multirow{3}{*}{ Informações } & 1. deve conter respostas de dúvidas mais frequentes. \\
\hline & 2. deve conter os locais que atendem pessoas do Crisdown. \\
\hline & 3. deve conter agenda com os eventos principais do Crisdown. \\
\hline \multirow[b]{2}{*}{ Localização } & 1. deve conter um mapa para chegar ao Crisdown. \\
\hline & $\begin{array}{l}\text { 2. deve conter no mapa indicação de atividades e atendimentos vinculados ao Crisdown próxima à localização } \\
\text { geográfica do usuário. }\end{array}$ \\
\hline \multirow[t]{2}{*}{ Fila de espera } & $\begin{array}{l}\text { 1. deve haver questionário para classificação de risco ambulatorial: Risco familiar, Risco psicossocial e risco de } \\
\text { saúde; estes riscos deve mostrar qual a prioridade daquele paciente e qual é a colocação na fila de espera de cada } \\
\text { especialidade. A classificação pode ser alterada de acordo coma situação do paciente. }\end{array}$ \\
\hline & 2. deve ter um contador da fila e o tempo estimado para atendimento em cada especialidade. \\
\hline \multirow{2}{*}{ Relatórios gerencias } & 1. deve gerar diversos relatórios gerenciais a respeito do aplicativo. \\
\hline & 2. deve gerar relatórios a respeito da fila de espera e dos atendimentos. \\
\hline
\end{tabular}

Fonte: Elaborado pela autora

\section{Conforme explicitado anteriormente requisitos não-funcionais, são restrições sobre os serviços} ou funções oferecidas pelo sistema. Diante disto, no Quadro 3 são expostos os requisitos nãofuncionais identificados.

Quadro 3 - Requisitos não-funcionais

\begin{tabular}{|c|c|c|}
\hline \multicolumn{3}{|r|}{ Requisitos não-funcionais identificados } \\
\hline \multirow{5}{*}{$\begin{array}{l}\text { Requisitos de } \\
\text { produto }\end{array}$} & Requisitos de facilidade de uso & Programa intuitivo, sem necessidade de aprimorar o conhecimento para utilizar. \\
\hline & \multirow{2}{*}{ Requisitos de eficiência } & De desempenho: a resposta do aplicativo deve ser rápida. \\
\hline & & de espaço: como será web, deverá ocupar \\
\hline & Requisitos de confiabilidade & Deve ter dis ponibilidade alta, evitando indis ponibilidade do sistema. \\
\hline & Requisitos de portabilidade & $\begin{array}{l}\text { O sistema deve rodar web, nos diversos navegadores de computador, bem como ter } \\
\text { adaptações para as plataformas Android e iOS. }\end{array}$ \\
\hline \multirow{3}{*}{$\begin{array}{c}\text { Requisitos } \\
\text { organizacionais }\end{array}$} & Requisitos de entrega & As definições de entrega devem ser previamente definidas pelo Cris down. \\
\hline & Requisitos de implementação & $\begin{array}{l}\text { O aplicativo deve ser desenvolvido em linguagens de programação adequada às } \\
\text { funcionalidades. }\end{array}$ \\
\hline & Requisitos de padrões & Os padrões devem seguir aqueles a serem estabelecidos pelo Crisdown \\
\hline \multirow{4}{*}{$\begin{array}{l}\text { Requisitos } \\
\text { externos }\end{array}$} & $\begin{array}{l}\text { Requisitos } \\
\text { interoperabilidade }\end{array}$ & $\begin{array}{l}\text { O aplicativo deve se comunicar com banco de dados externos, nenhuma informação deve ficar } \\
\text { vinculada ao computador ou outro tipo de aparelho. As informações devem estar em nuvem } \\
\text { segura. }\end{array}$ \\
\hline & Requisitos éticos & $\begin{array}{l}\text { O sistema não deve revelar quaisquer informações pessoais sobre os usuários do sistema ao } \\
\text { pessoal da biblioteca que usa o sistema, com exceção do nome e outros dados previamente } \\
\text { autorizados. }\end{array}$ \\
\hline & \multirow{2}{*}{ Requisitos legais } & de privacidade: Deverá atender às políticas de privacidade do usuário; \\
\hline & & de segurança: deverá atender todas as legis lações referentes à segurança das informações. \\
\hline
\end{tabular}




\subsection{CONCEITO DO PRODUTO}

Definiu-se a princípio que o aplicativo a ser desenvolvido seria web responsivo, ou seja, poderia ser utilizado tanto em formato de App quanto em formato Web. Assim, os usuários podem utilizar seus aparelhos móveis, como smartphones ou tablets, bem como computador. Por ser web, o aplicativo se torna mais vantajoso ao observar que os médicos do Crisdown necessitam inserir muitos dados. Desta forma, ao ter a possibilidade de utilizar o computador, facilita-se o trabalho além de tornar a inserção de informações mais confortável.

Outra vantagem de ser web é em relação à necessidade de contratação de profissional para o desenvolvimento do aplicativo, pois assim necessitará de apenas um profissional. Caso fosse optado por nativo, seria necessária a contratação de mais profissionais tendo em vista que a linguagem exigida para cada plataforma é diferente.

As funções do aplicativo estarão disponíveis de acordo com o perfil do usuário. Os perfis definidos são: paciente, familiares, profissionais de saúde e médicos do Crisdown.

Para os perfis de paciente e de familiares, serão disponibilizados: (1) dados do paciente, (2) direcionamento a jogos e aplicativos educacionais, (3) comunicação por meio de chat ou mensagem direta, (4) localização do Crisdown e de outros locais que podem ser úteis ao paciente, (5) agenda de consultas, (7) informações relevantes a respeito da Síndrome de Down e do Crisdown, bem como, perguntas e respostas mais frequentes.

Para o perfil de profissionais de saúde, haverá possibilidade de consultar o paciente pelo número de SES (apenas informações básicas e autorizadas), será possível inserir informações do atendimento realizado, bem como ter acesso às diversas funções acima citadas. O número de SES é a numeração fornecida pela Secretaria de Saúde do Distrito Federal para fins de registro e controle dos pacientes da rede pública de saúde.

Já com o perfil de médico do Crisdown, será possível acessar todas as funções acima citadas, além de poder modificar e/ou atualizar informações do paciente. Os médicos do Crisdown que serão responsáveis pelos questionamentos de avaliação do risco ambulatorial. Após o questionário realizado, o aplicativo, a partir dos parâmetros definidos pela equipe do Crisdown, indicará qual especialidade merece maior atenção para aquele paciente. Na mesma análise, o aplicativo indicará a classificação do paciente na fila de espera. 
Na Figura 5 é apresentada a visão geral do funcionamento do aplicativo, tornando o entendimento mais fácil. Conforme explicitado acima, são quatro tipos de perfis: paciente, familiares, profissionais de saúde e médicos do Crisdown. Desta maneira, cada perfil possui as funções que podem ser acessadas, conforme explicado acima.

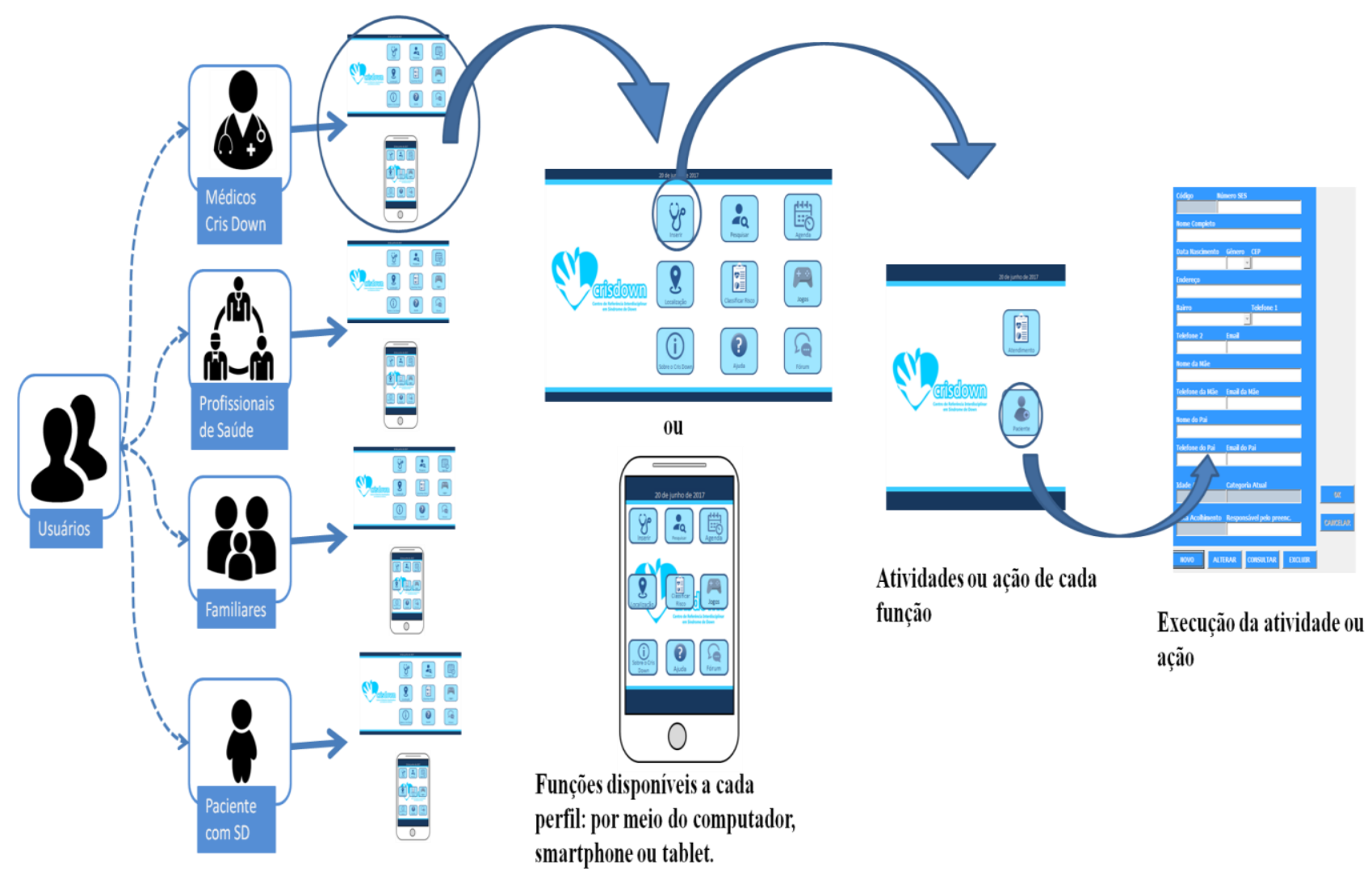

Figura 5 - Visão geral do aplicativo

Fonte: Elaborado pela autora

Vale ressaltar que existem dois aspectos que tornam o desenvolvimento deste aplicativo ainda mais importante: (1) a integração dos quatro tipos de usuários no mesmo aplicativo e, (2) o controle da fila de espera por meio da análise da classificação de riscos ambulatoriais. Estes pontos, até o presente momento, nunca foram explorados por aplicativo ou qualquer outro tipo de programa de computador para síndrome de down. Outro aspecto é a questão de linha do cuidado. Para torná-la visível, necessita de um sistema que viabilize as comunicações e as informações em toda linha. 
Ao analisar os outros aplicativos, observa-se que estes não possuem as funcionalidades necessárias para viabilizar a linha do cuidado. Os outros aplicativos também são de grande valia às pessoas com Síndrome de Down e aos familiares. No entanto, ter um aplicativo inovador que consiga conciliar todos interessados, contribui muito para o desenvolvimento das pessoas com SD e na linha do cuidado.

Para ilustrar melhor como esse valor é criado pelo cliente, foi montado um esquema que demonstra as funcionalidades que cada segmento de cliente poderá utilizar. Na Figura 6, é apresentada as funcionalidades que os médicos do Crisdown podem utilizar, é importante salientar que, com este perfil, pode realizar todos os tipos de pesquisas, modificações e inserção de pacientes e atendimentos.

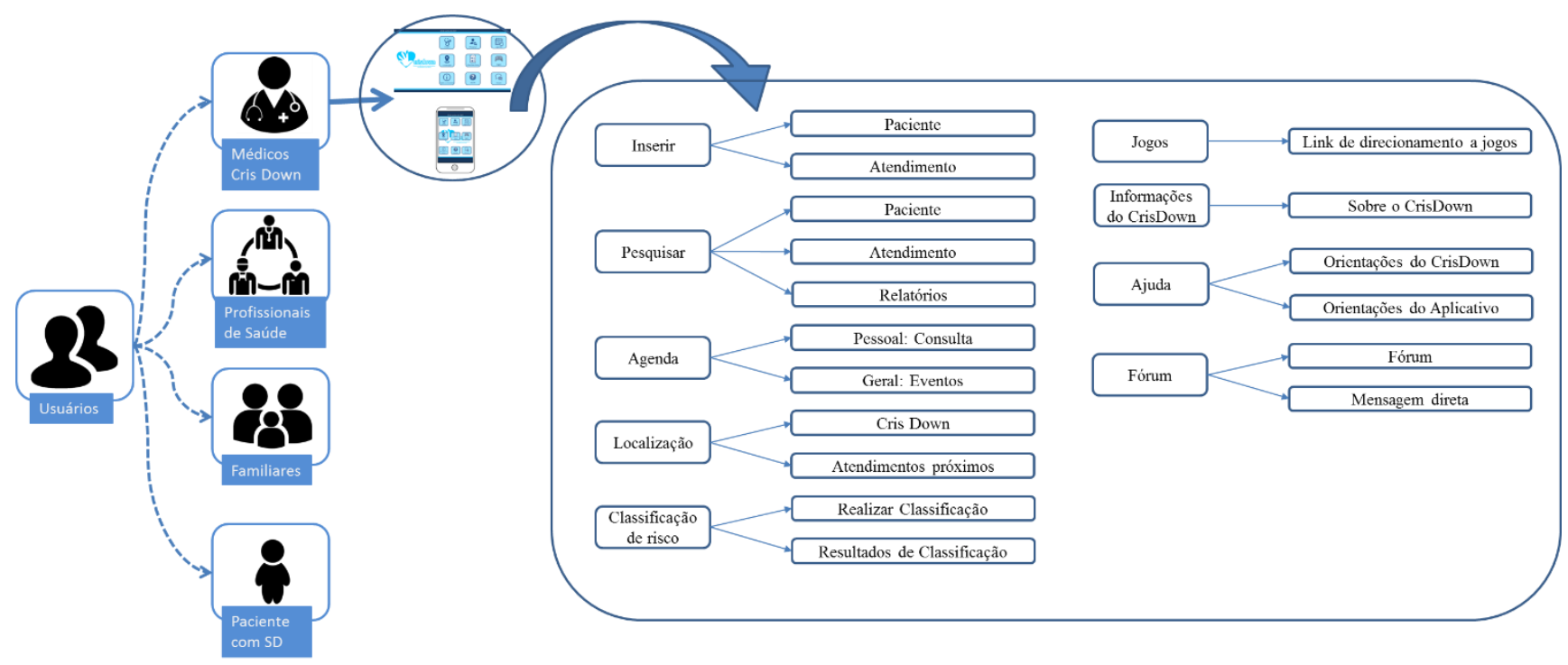

Figura 6 - Funcionalidades para os médicos do Crisdown

Fonte: Elaborado pela autora

Na Figura 7 são apresentadas as funcionalidades para o perfil de profissionais de saúde. Neste perfil não pode modificar nada referente ao paciente, pode inserir atendimento e realizar consulta de alguns dados importantes em relação ao paciente e ao atendimento. 


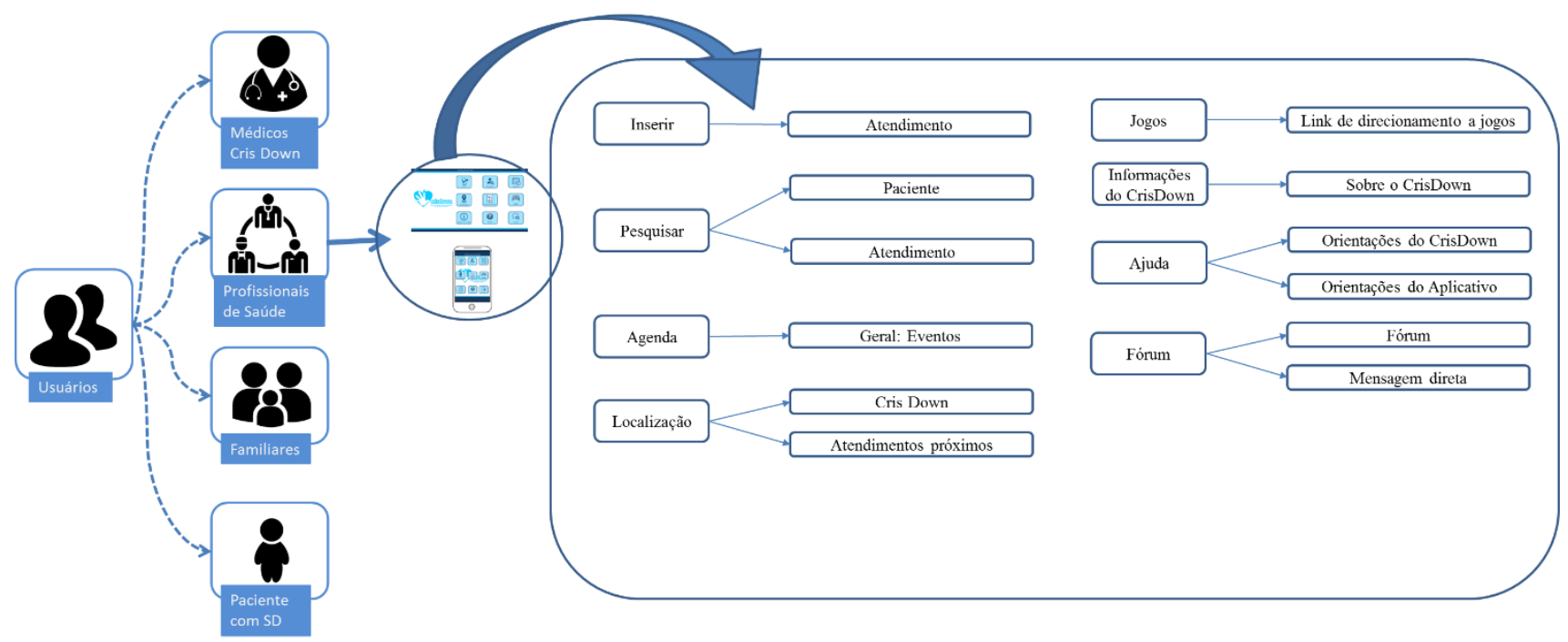

Figura 7- Funcionalidades para os profissionais de saúde

Fonte: Elaborado pela autora

Na Figura 8, é apresentada as funcionalidades para o perfil dos familiares. Neste perfil é possível inserir algumas informações básicas a respeito de um atendimento que o paciente tenha tido. Importante salientar que a pesquisa é apenas do paciente registrado como familiar.

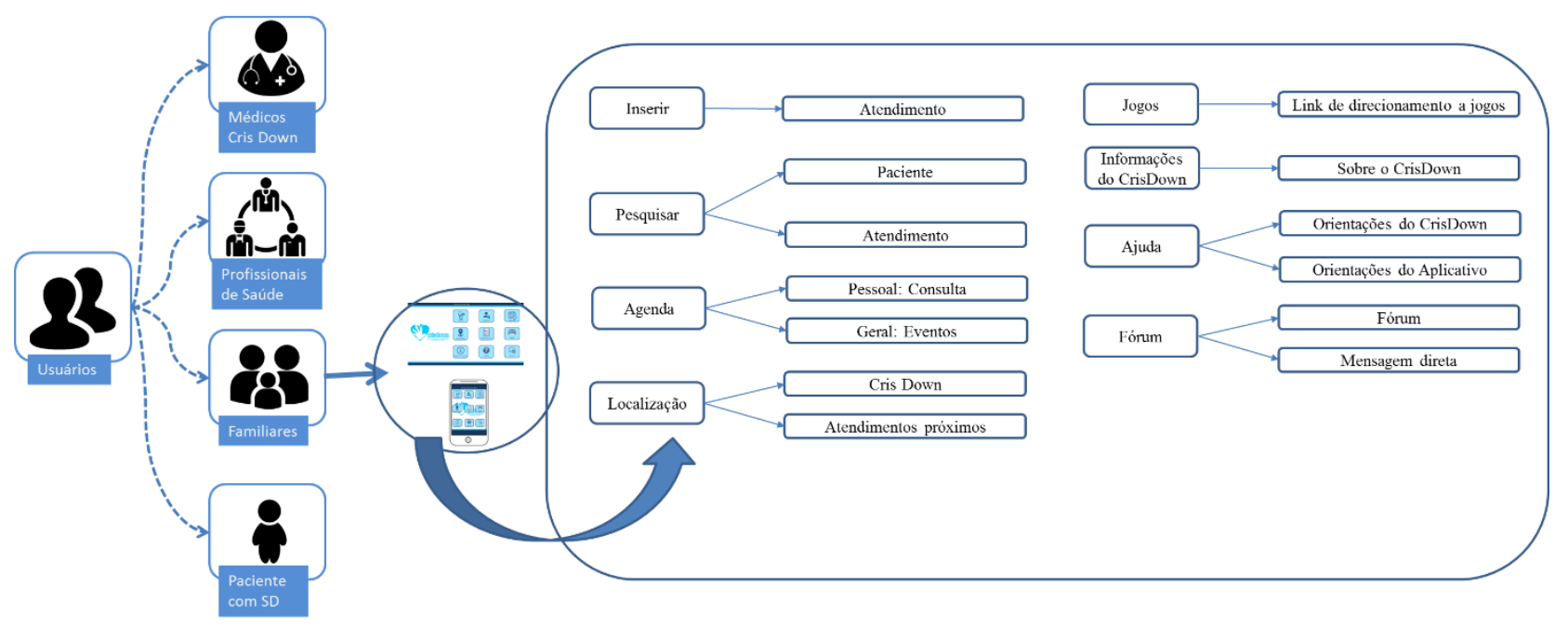

Figura 8 - Funcionalidades para os familiares

Fonte: Elaborado pela autora

Na Figura 9, é apresentada as funcionalidades para o perfil dos pacientes. Neste perfil é possível consultar os dados do paciente e dos atendimentos de maneira simplificada, ou seja, só algumas informações estarão disponíveis. Os jogos são direcionados para estes pacientes. 


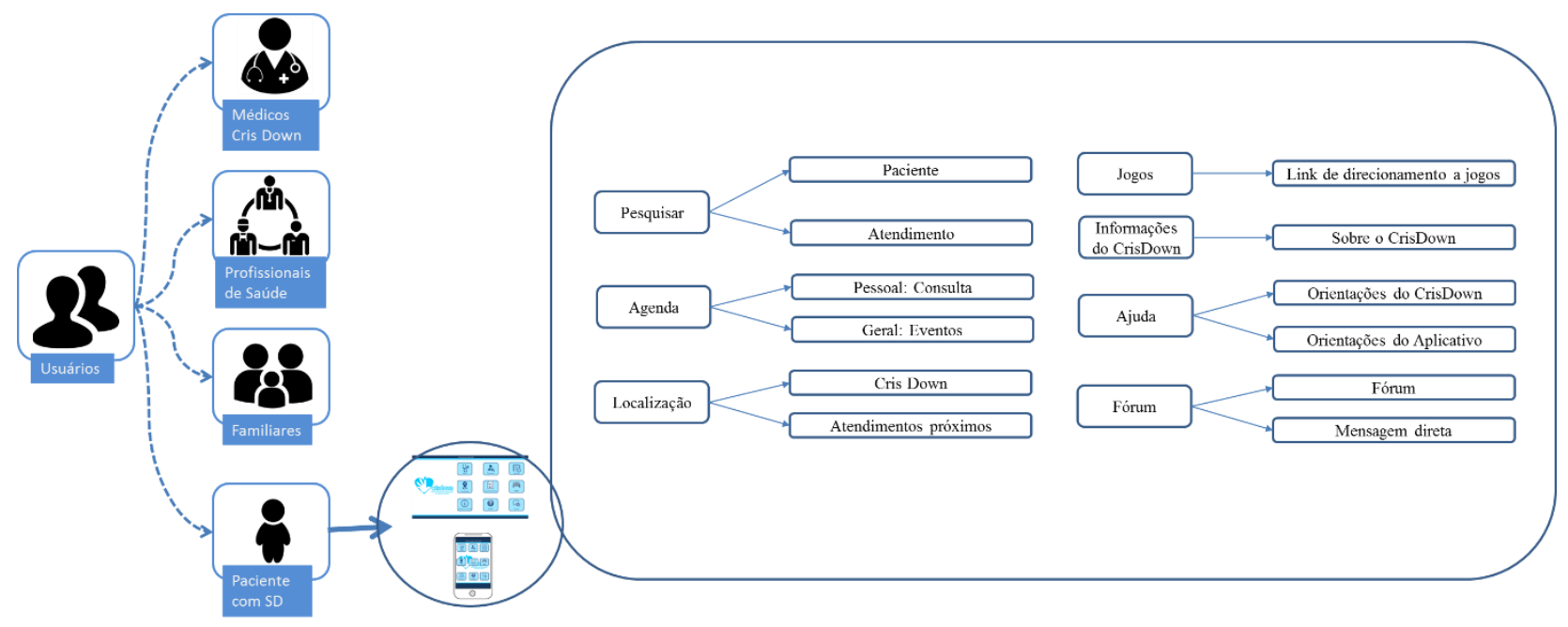

Figura 9 - Funcionalidades para os pacientes com SD

Fonte: Elaborado pela autora

Quanto à infraestrutura, estão inclusas as atividades-chaves, os recursos principais e parceiras principais. Nas atividades-chave contém a implementação e atualização do aplicativo, monitoramento, e estabelecer parcerias. Recursos principais incluem equipe desenvolvedora e investidores. As parcerias principais são associações, institutos e o governo.

$\mathrm{Na}$ viabilidade financeira contém a estrutura de custos e fontes de receita. Na estrutura de custos estão inclusos salários, custos fixos e variáveis. $\mathrm{Na}$ fonte de renda foram considerados investimentos de instituições voltadas à $\mathrm{SD}$, recursos governamentais, doações de pessoas físicas e fomento ou financiamento para pesquisa em inovação.

\section{CONSIDERAÇÕES FINAIS}

O projeto propõe o desenvolvimento de um aplicativo voltado à linha do cuidado às pessoas com Síndrome de Down, atualmente coordenado pelo Crisdown. Observou-se que o aplicativo tem potencial para o mercado e tem grande significado, tendo em vista a possibilidade de melhorar os atendimentos, a atenção e os cuidados com as pessoas que possuem Síndrome de Down.

Foi sugerida uma nova metodologia que alia os modelos de referência em PDP, proposto por Rozenfeld et al. (2006) e a Metodologia de Referência Mecatrônico - MRM proposta por Barbalho (2006) ao tipo de produto que estava por ser planejado. O produto é o desenvolvimento de aplicativo. Portanto, há várias peculiaridades que não são vislumbradas em produtos de manufatura. Neste sentido, para que atendesse aos requisitos dos clientes, foram 
realizadas algumas adaptações no modelo, contempladas aqui na fase de especificações do produto.

A fase de especificações do produto foi composta pela análise das reuniões realizadas com os principais clientes, definições de requisitos, conceito do produto e desenvolvimento de um plano de negócio. Com isto, foi possível identificar as reais necessidades dos clientes do aplicativo e definir especificações mais claras para o desenvolvedor do aplicativo. Quanto aos requisitos, estes foram levantados baseados na técnica proposta pela engenharia de requisitos.

Com base no desenvolvimento e nos resultados desse projeto, propõem-se os seguintes estudos futuros:

- Desenvolvimento do aplicativo proposto pelo presente projeto;

- Analisar a metodologia proposta neste projeto, e torná-la mais eficiente e eficaz para o levantamento de requisitos de aplicativos.

Levantar requisitos junto a um número maior de possíveis usuários.

\section{REFERÊNCIAS}

BARBALHO, S. C. M. Modelo de referência para o desenvolvimento de produtos mecatrônicos: proposta e aplicações. Escola de Engenharia de São Carlos. São Carlos: s.n., 2006. p. 257, Tese (Doutorado em Engenharia Mecânica).

Barbalho, S. C. M.; Rozenfeld, H. Modelo de referência para o processo de desenvolvimento de produtos mecatrônicos (MRM): validação e resultados de uso. Gestão \& Produção, v. 20, p. 162-179, 2013.

DÉA, V. H. S. D.; DÉA, V. P. B. D. Informações gerais sobre a síndrome de Down. 2009.

FONSECA, J. J. S. Metodologia da pesquisa científica. Fortaleza: UEC, 2002.

PRESSMAN, R.; MAXIM, B. Engenharia de software: Uma abordagem profissional. 8 ed. São Paulo: Amgh Editora, 2016. 968 p.

VOIVODIC, M. A.; STORER, M. R. de S. O desenvolvimento cognitivo das crianças com síndrome de Down à luz das relações familiares. Psicologia: teoria e prática, São Paulo, p. 3140, nov. 2002.

ZAVE, Pamela. Classification of Research Efforts in Requirements Engineering. ACM Computing Surveys. v. 29, n. 4. Dev. 1997. p. 315-321. 
SOMMERVILlE, I. Engenharia de Software. 8. ed. São Paulo: Pearson Addison. Wesley, 2007. 